\title{
PERIODIC PHENOMENA IN THE CLASSICAL ADAMS SPECTRAL SEQUENCE
}

\author{
MARK MAHOWALD AND PAUL SHICK
}

\begin{abstract}
We investigate certain periodic phenomena in the classical Adams sepctral sequence which are related to the polynomial generators $v_{n}$ in $\pi_{*}(\mathrm{BP})$. We define the notion of a class $a$ in $\operatorname{Ext}_{A}(\mathbf{Z} / 2, \mathbf{Z} / 2)$ being $v_{n}$-periodic or $v_{n}$-torsion and prove that classes that are $v_{n}$-torsion are also $v_{k}$-torsion for all $k$ such that $0 \leqslant k \leqslant n$. This allows us to define a chromatic filtration of $\operatorname{Ext}_{A}(\mathbf{Z} / 2, \mathbf{Z} / 2)$ paralleling the chromatic filtration of the Novikov spectral sequence $E_{2}$-term given in $[13]$.
\end{abstract}

1. Introduction and statement of results. This work is motivated by a desire to understand something of the periodic phenomena noticed by Miller, Ravenel and Wilson in their work on the Novikov spectral sequence from the point of view of the classical Adams spectral sequence. The $E_{2}$-term of the classical Adams spectral sequence (hereafter abbreviated clASS) is isomorphic to $\operatorname{Ext}_{A}(\mathbf{Z} / 2, \mathbf{Z} / 2)$, where $A$ is the mod 2 Steenrod algebra. This has been calculated completely in the range $t-s \leqslant 70$ [17]. The stem-by-stem calculation is quite tedious, though, so one looks for more global sorts of phenomena. The first result in this direction was the discovery of a periodic family in $\pi_{*}\left(S^{0}\right)$ and their representatives in $\operatorname{Ext}_{A}(\mathbf{Z} / 2, \mathbf{Z} / 2)$, discussed by Adams in [2] and by Barratt in [4]. This stable family, which is present for all primes $p$, is often denoted by $\left\{\boldsymbol{\alpha}_{t}\right\}$ and is thought of as $v_{1}$-periodic, where $v_{1}$ is the polynomial generator of degree $2(p-1)$ in $\pi_{*}(\mathrm{BP})=\mathbf{Z}_{(p)}\left[v_{1}, v_{2}, \ldots\right]$. Using the Novikov spectral sequence based on the spectrum BP, the families $\left\{\beta_{t}\right\}$ and $\left\{\gamma_{t}\right\}$ have been investigated for sufficiently large odd primes [13]. These are $v_{2^{-}}$and $v_{3}$-periodic families, respectively. In [6 and 10], a start was made toward understanding these $v_{i}$-periodic families from the point of view of the clASS. Here we continue this effort, defining the concepts of $v_{i}$-peridocity and $v_{i}$-torsion in $\operatorname{Ext}_{A}(\mathbf{Z} / 2, \mathbf{Z} / 2)$ for all $i$.

Our method of study is to utilize certain Hopf subalgebras of $A$. Let $A_{n}$ denote the Hopf subalgebra generated by $\left(\mathrm{Sq}^{0}, \mathrm{Sq}^{1}, \mathrm{Sq}^{2}, \ldots, \mathrm{Sq}^{2^{\prime \prime}}\right)$. Then $\operatorname{Ext}_{A}(\mathbf{Z} / 2, \mathbf{Z} / 2)$ $\approx \lim _{k} \operatorname{Ext}_{A_{k}}(\mathbf{Z} / 2, \mathbf{Z} / 2)$. Our first result is:

TheOReM A. For $i$ any positive integer, there exists a unique nonzero divisor

$$
w_{i} \in \operatorname{Ext}_{A_{i}}^{2^{i+1}, 2^{i+1}\left(2^{1+1}-1\right)}(\mathbf{Z} / 2, \mathbf{Z} / 2)
$$

Received by the editors January 11, 1985 and in revised form, July 16, 1985 and April 15, 1986.

1980 Mathematics Subject Classification (1985 Revision). Primary 55T15, 55Q45; Secondary 55S10, $55 \mathrm{~N} 22$. 
such that $w_{i}$ restricts nontrivially to $\operatorname{Ext}_{E\left[Q_{1}\right]}(\mathbf{Z} / 2, \mathbf{Z} / 2)$, corresponding to the class $v_{i}^{2^{i+1}} \in \pi_{*}(\mathrm{BP})$.

We hereafter denote $w_{i}$ by $v_{i}^{2^{i+1}} \in \operatorname{Ext}_{A_{i}}(\mathbf{Z} / 2, \mathbf{Z} / 2)$. For $k>i$, there is also some power of $v_{i}$ present. In fact, we have the following:

THEOREM B. For $k$ any positive integer, there exist positive integers $N_{1}, N_{2}, \ldots, N_{k}$ such that

$$
\mathbf{Z} / 2\left[h_{0}, v_{1}^{\left(4 N_{1}\right)}, v_{2}^{\left(8 N_{2}\right)}, \ldots, v_{i}^{\left(2^{i+1} N_{i}\right)}, \ldots, v_{k}^{\left(2^{k+1} N_{h}\right)}\right] \subset \operatorname{Ext}_{A_{k}}(\mathbf{Z} / 2, \mathbf{Z} / 2) .
$$

Note that $N_{i}$ also depends on the value of $k$. Note aiso that $N_{k}$ can be chosen to be 1 by Theorem $\mathrm{A}$. In particular, Theorem $\mathrm{B}$ implies that for all $k \geqslant i$, some power of $v_{i}^{2^{i+1}}$ is present in $\operatorname{Ext}_{A_{k}}(\mathbf{Z} / 2, \mathbf{Z} / 2)$, with all of its powers nontrivial. For $k>i$, this choice of $v_{i}^{N}$ is not unique. For each $k \geqslant i$ we localize $\operatorname{Ext}_{A_{k}}(\mathbf{Z} / 2, \mathbf{Z} / 2)$ with respect to $v_{i}$. This gives a map

$$
f_{i}: \operatorname{Ext}_{A}(\mathbf{Z} / 2, \mathbf{Z} / 2) \rightarrow \underset{k}{\lim }\left[\operatorname{Ext}_{A_{k}}(\mathbf{Z} / 2, \mathbf{Z} / 2)\left(v_{i}^{-1}\right)\right],
$$

which enables us to define the following concept.

Definition (3.8). $x \in \operatorname{Ext}_{A}(\mathbf{Z} / 2, \mathbf{Z} / 2)$ is $v_{i}$-periodic if $f_{i}(x) \neq 0$, and is $v_{i}$-torsion otherwise.

Notice that the above definition is equivalent to the following: if $q_{k}^{*} \operatorname{Ext}_{A}(\mathbf{Z} / 2, \mathbf{Z} / 2) \rightarrow \operatorname{Ext}_{A_{k}}(\mathbf{Z} / 2, \mathbf{Z} / 2)$ denotes the natural projection, then $x \in$ $\operatorname{Ext}_{A}(\mathbf{Z} / 2, \mathbf{Z} / 2)$ is $v_{i}$-periodic if there exists a $K>0$ such that $q_{k}^{*}(x)\left(v_{i}^{2^{i+1} N_{i}}\right)^{s} \neq 0$ for all $s \geqslant 0$, for all $k \geqslant K$.

Our main theorem is

Theorem C. If $x \in \operatorname{Ext}_{A}(\mathbf{Z} / 2, \mathbf{Z} / 2)$ is $v_{n}$-periodic, then $x$ is also $v_{n+k}$-periodic for all $k \geqslant 0$.

Equivalent, if $x \in \operatorname{Ext}_{A}(\mathbf{Z} / 2, \mathbf{Z} / 2)$ is $v_{n}$-torsion, then $x$ is also $v_{k}$-torsion for all $k$ such that $0 \leqslant k \leqslant n$.

Analogous results are known for elements $x \in M$, where $M$ is a $\mathrm{BP}_{*} \mathrm{BP}$-comodule [9]. Our proof of Theorem $C$ is a simplified version of Johnson and Yosimura's proof of the BP-setting result.

Theorem $\mathrm{C}$ allows us to define a filtration

COROLlaRY D. There is a filtration, which we call the chromatic filtration,

$$
\operatorname{Ext}_{A}(\mathbf{Z} / 2, \mathbf{Z} / 2)=F_{-1} \supset F_{0} \supset F_{1} \supset \cdots \supset F_{i} \supset \cdots
$$

such that $F_{i}-F_{i+1}$ is the set of classes that are $v_{i+1}$-periodic but $v_{k}$-torsion for all $k \leqslant i$.

This paper is organized as follows. In $\S 2$, we construct our basic took, which is used for calculating Ext-groups. It is a variant of the Koszul resolution. In §3, we use this resolution to produce the periodicity elements of Theorem $\mathrm{A}$. We also prove 
Theorem B and develop the concept of $v_{i}$-periodicity in $\operatorname{Ext}_{A}(\mathbf{Z} / 2, \mathbf{Z} / 2)$. In $\S 4$, we construct certain operations

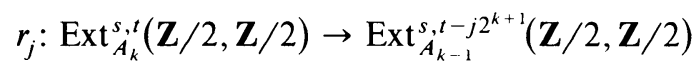

for $k \geqslant 1$, and state their basic properties. These are related to a certain decomposition of $A / / A_{k}$ given in [11]. Finally, in $\S 5$, we use these operations to prove Theorem $\mathrm{C}$ and deduce Corollary $\mathrm{D}$ from it.

Throughout the paper, we use cohomology with $\mathbf{Z} / 2$ coefficients. By "space", we mean a connective spectrum localized at the prime 2 . Odd primary analogs of these results are known, and will be discussed elsewhere. These results form the basis of the first chapter of the second author's Ph.D. thesis, completed at Northwestern University in 1984. We would like to thank Wolfgang Lellmann, Ralph Cohen and Mike Hopkins for many helpful discussions. We also thank the referee for his helpful comments and for pointing out an error in the original proof.

2. Koszul-type resolutions for calculating Ext-groups. In this section, we develop the machinery necessary to produce the periodicity elements in $\operatorname{Ext}_{A_{i}}(\mathbf{Z} / 2, \mathbf{Z} / 2)$ for $i \geqslant 1$. The basic tool used is a variant of the Koszul resolution [8] in which one "resolves" a polynomial algebra using an exterior algebra. A more concise account of this material appears in [7].

We begin by constructing the Koszul resolution complex. This will be an exact sequence to which the functor $\operatorname{Ext}_{A_{i}}(-, \mathbf{Z} / 2)$ will be applied to get a spectral sequence. We recall that the dual of the Steenrod algebra, $A^{*}$, is a polynomial algebra $\mathbf{Z} / 2\left[\xi_{1}, \xi_{2}, \ldots\right]$, where the degree of $\xi_{i}$ is $2^{i}-1$. Note that $A^{*}$ is both a right and left module over $A$, with the actions given by $\mathrm{Sq}\left(\xi_{k}\right)=\xi_{k}+\xi_{k-1}^{2}$ and $\left(\xi_{k}\right) \mathrm{Sq}=$ $\xi_{k}+\xi_{k-1}$, where $\mathrm{Sq}=\sum \mathrm{Sq}^{i}$. It is shown in [14] that $\chi\left(A / / A_{j}\right)^{*} \cong$ $\mathbf{Z} / 2\left[\xi_{1}^{2 j+1}, \xi_{2}^{2^{j}}, \ldots, \xi_{j+1}^{2}, \xi_{j+2}, \xi_{j+3}, \ldots\right]$, where $\chi$ denotes the canonical antiautomorphism of the Steenrod algebra and $A / / A_{j}$ denotes $A \otimes_{A_{j}} \mathbf{Z} / 2$. This isomorphism is one of algebras and left $A$-modules, where the left $A$-action on the polynomial algebra is given by the above formula, extended by the Cartan formula. This result generalizes to show that $\chi\left(A_{i} / / A_{i-1}\right)^{*} \cong E\left(\xi_{1}^{2^{i}}, \xi_{2}^{2^{i-1}}, \ldots, \xi_{j+1}\right)$, both as algebras and as left $A_{i}$-modules with the above $A_{i}$-action. If we denote $\chi\left(\xi_{k}\right)$ by $\zeta_{k}$, then we see that $\left(A_{i} /,^{\prime} A_{i-1}\right)^{*} \cong E\left(\zeta_{1}^{2^{i}}, \zeta_{2}^{2^{i-1}}, \ldots, \zeta_{i+1}\right)$, with the $A_{i}$-action now being given on the right: $\zeta_{i+1-j}^{2^{k}} \mathrm{Sq}^{2^{k}}=\zeta_{i-j}^{2^{k+1}}$ and $\zeta_{1}^{2^{i}} \mathrm{Sq}^{2^{i}}=1$, extended by the Cartan formula. For convenience, we denote the exterior algebra $\left(A_{i} / / A_{i-1}\right)^{*}$ by $E(i)$. It is important to note that $E(i)$ is an $A_{i}$-module but not an $A_{i-1}$-module. For example, $\left(A_{1} / / A_{0}\right)^{*}$ $\approx E\left(\zeta_{1}^{2}, \zeta_{2}\right)$ cannot be an $A$-module since $\mathrm{Sq}^{2} \mathrm{Sq}^{1} \mathrm{Sq}^{2}$ is nonzero on the top class $\zeta_{1}^{2} \zeta_{2}$. By the Adem relations, $\mathrm{Sq}^{2} \mathrm{Sq}^{1} \mathrm{Sq}^{2}=\mathrm{Sq}^{1} \mathrm{Sq}^{4}+\mathrm{Sq}^{4} \mathrm{Sq}^{1}$, so that if $E(1) \cong$ $\left(A_{1} / / A_{0}\right)^{*}$, it must have a nonzero class of degree 1 or 4 , which it does not.

As an $A_{i-1}$-module, we can decompose $E(i)$ into a direct sum: $E(i) \cong$ $\oplus_{\sigma \geqslant 0} E_{\sigma}(i)$, where $E_{\sigma}(i)$ is given as a $\mathbf{Z} / 2$-vector space as the span of monomials of length $\sigma, x_{1} x_{2} \cdots x_{\sigma}$, where each $x_{j} \in\left(\zeta_{1}^{2^{i}}, \zeta_{2}^{2^{i-1}}, \ldots, \zeta_{i+1}\right)$ and $x_{j} \neq x_{k}$ for $j \neq k$. Each of these $E_{\sigma}(i)$ 's is closed under the $A_{i-1}$-action inherited from $E(i)$ and is also an $A$-module. 
We now define the polynomial algebra we will use to resolve $E(i)$. Let $R(i)=$ $\mathbf{Z} / 2\left[\zeta_{1}^{2^{\prime}}, \zeta_{2}^{2^{i-1}}, \ldots, \zeta_{i+1}\right]$, the graded polynomial algebra on generators $\zeta_{1}^{2^{\prime}}$, $\zeta_{2}^{2^{i-1}}, \ldots, \zeta_{i+1}$. This is an $A$-module, with right action given by $\zeta_{i+1-j}^{2^{k}} \mathrm{Sq}^{2^{k}}=\zeta_{i-j}^{2^{k+1}}$ and $\zeta_{1}^{2^{i}} \mathrm{Sq}^{2^{i}}=1$, extended by the Cartan formula. If we consider just the $A_{i-1}$-module structure that this imposes on $R(i)$, then we can decompose this into a direct sum: $R(i) \cong \bigoplus_{\sigma \geqslant 0} R_{\sigma}(i)$. Here, $R_{\sigma}(i)$ is given as a $\mathbf{Z} / 2$-vector space as the span of monomials of length $\sigma$ in $\left(\zeta_{1}^{2^{i}}, \zeta_{2}^{2^{i-1}}, \ldots, \zeta_{i+1}\right)$. Each of the $R_{\sigma}(i)$ 's is a separate $A$-module.

To construct our resolution, we form the right $A_{i}$-modules $E_{r}(i) \stackrel{\vec{J}}{\otimes_{\mathbf{z} / 2}} R_{s}(i)$ where $r, s>0$. Here " $\stackrel{\Delta}{\mathbf{Z} / 2}$ " means tensoring over $\mathbf{Z} / 2$ with the $A_{i}$-action given by the Cartan formula. Actually, each of these $E_{r} \otimes_{\mathbf{z} / 2} R_{s}$ 's is an $A$-module, but we need only the $A_{i}$-module structure. We construct maps $k_{r, s}: E_{r} \otimes_{\mathrm{z} / 2} R_{s} \rightarrow E_{r-1}$ $\otimes_{\mathbf{z} / 2} R_{s+1}$ by

$$
k_{r, s}\left(x_{1} x_{2} \cdots x_{r} \otimes p\right)=\sum_{j=1}^{r} x_{1} \cdots \hat{x}_{j} \cdots x_{r} \otimes x_{j} p, \quad \text { for all } r \geqslant 1, s \geqslant 0 .
$$

To see that these are $A_{i}$-maps, consider

$$
k_{r, s}\left[\left(x_{1} x_{2} \cdots x_{r} \otimes p\right) \mathrm{Sq}^{m}\right]=k_{r, s}\left[\sum_{M}\left(x_{b_{1}} x_{b_{2}} \cdots x_{b_{r}}\right) \otimes p \mathrm{Sq}^{\left(m-\sum a_{t}\right)}\right]
$$

where $M$ runs through the set of all sequences $\left(a_{1}, \ldots, a_{r}\right)$ such that $x_{t} \mathrm{Sq}^{a_{t}}=x_{b_{i}}$. Evaluating $k_{r, s}$ on this, we get

$$
\sum_{j, M}\left[\left(x_{b_{1}} x_{b_{2}} \cdots \hat{x}_{b_{j}} \cdots x_{b_{r}}\right) \otimes x_{b_{j}} p \mathrm{Sq}^{\left(m-\sum a_{t}\right)}\right],
$$

which is exactly $\left[k_{r, s}\left(x_{1} x_{2} \cdots x_{r} \otimes p\right)\right] \mathrm{Sq}^{m}$. We compose these $A_{i}$-module maps into a sequence, recalling that $E_{r}=0$ for $r>i+1$ :

$$
0 \rightarrow E_{i+1} \otimes R_{s} \rightarrow E_{i} \otimes R_{s+1} \rightarrow \cdots \rightarrow E_{0} \otimes R_{s+i+1} \rightarrow 0
$$

These sequences are exact, as one can check, although this is quite tedious. We can get around this by summing the sequences over a constant $s$ :

$$
\begin{aligned}
& \dot{0} \quad \rightarrow \quad E_{i+1} \otimes R_{s+2} \rightarrow \cdots \\
& 0 \quad \rightarrow \quad E_{i+1} \otimes R_{s+1} \rightarrow E_{i} \otimes R_{s+2} \quad \rightarrow \cdots \\
& 0 \quad \rightarrow \quad E_{i+1} \otimes R_{s} \rightarrow E_{i} \otimes R_{s+1} \quad \rightarrow \quad E_{i-1} \otimes R_{s+2} \rightarrow \cdots \\
& 0 \rightarrow E_{i+1} \otimes R_{s-1} \rightarrow E_{i} \otimes R_{s} \rightarrow E_{i-1} \otimes R_{s+1} \rightarrow E_{i-1} \otimes R_{s+2} \rightarrow \cdots \\
& \therefore \rightarrow E_{2} \otimes R_{s-1} \rightarrow E_{1} \otimes R_{s} \rightarrow E_{0} \otimes R_{s+1} \quad \rightarrow \quad \rightarrow \quad 0 \\
& \cdots \rightarrow E_{1} \otimes R_{s-1} \quad \rightarrow \quad E_{0} \otimes R_{s} \quad \rightarrow \quad 0 \\
& \cdots \rightarrow E_{0} \otimes R_{s-1} \quad \rightarrow \quad 0 \\
& \ldots \rightarrow 0
\end{aligned}
$$

The result is a sequence of $A_{i}$-modules:

$$
0 \rightarrow \mathbf{Z} / 2 \rightarrow E(i) \otimes R_{0}(i) \rightarrow E(i) \otimes R_{1}(i) \rightarrow \cdots
$$


which is exact by the classical result of Koszul. The differential is given by

$$
\partial_{\sigma}\left[\left(x_{1} x_{2} \cdots x_{r}\right) \otimes p\right]=\sum_{j=1}^{r}\left(x_{1} \cdots \hat{x}_{j} \cdots x_{r}\right) \otimes x_{j} p .
$$

Denote the dual of $R_{\sigma}(i)$ by $N_{\sigma}(i)$. Then, dualizing the exact sequence of right $A_{i}$-modules in (2.1), we obtain

LEMma (2.2). The sequence

$$
0 \leftarrow \mathbf{Z} / 2 \stackrel{\varepsilon}{\leftarrow} A_{i} / / A_{i-1} \otimes N_{0}(i) \stackrel{\partial_{0}}{\leftarrow} \ldots \stackrel{\partial_{\sigma-1}}{\leftarrow} A_{i} / / A_{i-1} \otimes N_{\sigma}(i) \stackrel{\partial_{\sigma}}{\leftarrow} \ldots
$$

is exact as a sequence of left $A_{i}$-modules.

We need the following lemma.

LemMA (2.3). For any $A_{i}$-module $M, A_{i} / / A_{i-1} \stackrel{\Delta}{\otimes}{ }_{\mathrm{z} / 2} M \cong A_{i} \stackrel{L}{\otimes}_{A_{i-1}} M$, as left $A_{i}$-modules, where " $\stackrel{L}{\otimes_{A_{i-1}}}$ " means tensor over $A_{i-1}$ with the $A_{i}$-action taken on the left factor.

A proof of this lemma can be found in [19].

We have now completed the proof of the following result.

THEOREM (2.4). For the family of $A$-modules $N_{\sigma}(i), \sigma \geqslant 0$, and $A_{i}$-maps $\partial_{\sigma}$ : $A_{i} \otimes_{A_{i-1}} N_{\sigma+1}(i) \rightarrow A_{i} \otimes_{A_{i-1}} N_{\sigma}(i)$ defined above, the sequence

$$
0 \leftarrow \mathbf{Z} / 2 \stackrel{\varepsilon}{\leftarrow} A_{i} \otimes_{A_{i-1}} N_{0}(i) \stackrel{\partial_{0}}{\leftarrow} A_{i} \otimes_{A_{i-1}} N_{1}(i) \stackrel{\partial_{1}}{\leftarrow} \ldots \stackrel{\partial_{\sigma-1}}{\leftarrow} A_{i} \otimes_{A_{i-1}} N_{\sigma}(i) \stackrel{\partial_{\sigma}}{\leftarrow} \ldots
$$

is exact as a sequence of $A_{i}$-modules.

We refer to this as the Koszul-type resolution for $\mathbf{Z} / 2$ over $A_{i}\left(K R_{i}\right.$ or $K R$ if $i$ is understood).

Also as an easy consequence of 2.4 we have

Corollary (2.5). For $M$ any left $A_{i}$-module, the complex

$$
0 \leftarrow M \stackrel{\varepsilon}{\leftarrow} A_{i} \otimes_{A_{i-1}} N_{0}(i) \otimes_{\mathbf{z} / 2} M \stackrel{\partial_{0}}{\leftarrow} \cdots \stackrel{\partial_{\sigma-1}}{\leftarrow} A_{i} \otimes_{A_{i-1}} N_{\sigma}(i) \otimes_{\mathbf{z} / 2} M \stackrel{\partial_{\sigma}}{\leftarrow} \cdots
$$

is exact $\left(K R_{i}(M)\right)$.

The usefulness of such resolutions is that one can apply various functors to them to obtain spectral sequences. Our goal is to produce a spectral sequence converging to $\operatorname{Ext}_{A_{i}}(M, \mathbf{Z} / 2)$ for $M$ an $A_{i}$-module. To this end, we divide the complex of (2.4) into short exact sequences:

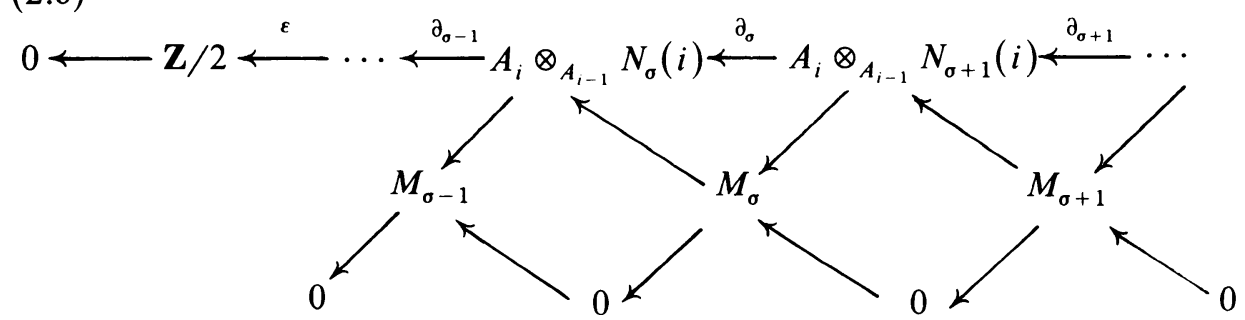


We apply the functor $\operatorname{Ext}_{A_{i}}^{s-\sigma, t}(-, \mathbf{Z} / 2)$ to (2.6). This associates to each short exact sequence $0 \rightarrow M_{\sigma} \rightarrow A_{i} \otimes_{A_{i-1}} N_{\sigma} \rightarrow M_{\sigma-1} \rightarrow 0$ a long exact sequence:

$$
\begin{aligned}
\cdots & \stackrel{i}{\rightarrow} \operatorname{Ext}_{A_{i}}^{s-\sigma, t}\left(M_{\sigma-1}, \mathbf{Z} / 2\right) \stackrel{j}{\rightarrow} \operatorname{Ext}_{A_{i}}^{s-\sigma, t}\left(A_{i} \otimes_{A_{i-1}} N_{\sigma}, \mathbf{Z} / 2\right) \\
& \stackrel{k}{\rightarrow} \operatorname{Ext}_{A_{i}}^{s-\sigma, t}\left(M_{\sigma}, \mathbf{Z} / 2\right) \stackrel{i}{\rightarrow} \operatorname{Ext}_{A_{i}}^{s-\sigma+1, t}\left(M_{\sigma-1}, \mathbf{Z} / 2\right) \stackrel{j}{\rightarrow} \cdots
\end{aligned}
$$

We fit these long exact sequences together to form an exact couple:

$$
\begin{aligned}
& D_{1}^{\sigma, s, t}=\operatorname{Ext}_{A_{i}}^{s-\sigma, t}\left(M_{\sigma-1}, \mathbf{Z} / 2\right), \\
& E_{1}^{\sigma, s, t}=\operatorname{Ext}_{A_{i}}^{s-\sigma, t}\left(A_{i} \otimes_{A_{i-1}} N_{\sigma}, \mathbf{Z} / 2\right) \cong \operatorname{Ext}_{A_{i-1}}^{s-\sigma, t}\left(N_{\sigma}, \mathbf{Z} / 2\right)
\end{aligned}
$$

by the change of rings isomorphism. The maps in the long exact sequence (2.7) give the maps in the exact couple

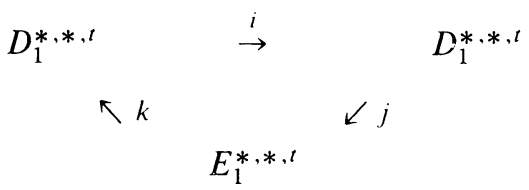

These maps have the following trigradings:

$$
\begin{array}{cc} 
& \sigma s t \\
i: & (-1,0,0) \\
j: & (+1,+1,0) \\
k: & (0,0,0)
\end{array}
$$

Thus, $d_{r}: E_{r}^{\sigma, s, t} \rightarrow E_{r}^{\sigma+r, s+1, t}$.

To see to what the spectral sequence converges, one forms a double complex, taking a projective resolution of each term of the complex (2.4). The resulting Grothendieck-type spectral sequence clearly converges to $\operatorname{Ext}_{A_{i}}(\mathbf{Z} / 2, \mathbf{Z} / 2)$. This completes the proof of the following result.

THEOREM (2.8). For $i$ any positive integer, there is a family of $A$-modules, $N_{\sigma}(i)$, $\sigma \geqslant 0$, defined above, such that for any $A_{i}$-module $M$ there is a trigraded spectral sequence converging to $\operatorname{Ext}_{A_{i}}^{s, t}(M, \mathbf{Z} / 2)$, with

$$
E_{1}^{\sigma, s, t} \cong \operatorname{Ext}_{A_{t-1}}^{s-\sigma, t}\left(N_{\sigma}(i) \otimes M, \mathbf{Z} / 2\right) \text {. }
$$

This is called the Koszul spectral sequence for $M$ over $A_{i}\left(\operatorname{KSS}_{i}(M)\right)$. Note that a trigraded spectral sequence is a family of spectral sequences, one for each $t$.

Theorem (2.8) allows us to compute $\operatorname{Ext}_{A_{i}}(M, \mathbf{Z} / 2)$ in terms of

$$
\operatorname{Ext}_{A_{i-1}}\left(N_{\sigma} \otimes M, \mathbf{Z} / 2\right) \text {. }
$$

This makes calculation of $\operatorname{Ext}_{A_{1}}(M, \mathbf{Z} / 2)$ very easy since $\operatorname{Ext}_{A_{0}}(-, \mathbf{Z} / 2)$ is quite simple to compute. $\operatorname{Ext}_{A_{2}}(M, \mathbf{Z} / 2)$ is also fairly tractable for reasonable $A_{2}$-modules $M$, as seen in [7], where $\operatorname{Ext}_{A_{2}}\left(H^{*} \mathbf{R} P_{N}^{\infty}, \mathbf{Z} / 2\right)$ is calculated for all $N$. One should note that the $d_{1}$-differentials in the KSS are induced from the maps $\partial_{\sigma}$ of the complex (2.4). These are $A_{i}$-maps, but are not extended $A_{i-1}$-maps. That is, $\partial_{\sigma}$ is not given as $\operatorname{id}_{A_{i}} \otimes\left(N_{\sigma} \stackrel{f}{\leftarrow} N_{\sigma+1}\right)$ for any $A_{i-1}$-map $f$. Thus the $d_{1}$-differential in the KSS need not respect the Yoneda product structure in $\operatorname{Ext}_{A_{i}}(-, \mathbf{Z} / 2)$, although there is a product present. 
We conclude this section with an easy proof of the well-known "ledge theorem."

Theorem (2.9) ("Ledge Theorem"). Let $M$ be a finite $A_{i}$-module such that $M_{r}=0$ for $r>m$. Then $\operatorname{Ext}_{\dot{A}_{i}}^{s, t}(M, \mathbf{Z} / 2)=0$ for $t-s>\left(2^{i+1}-2\right) s+m$.

Proof. We use induction on $i$, with the initial case, $i=1$, clear from calculating by a minimal resolution. We assume that $\operatorname{Ext}_{A_{i-1}}^{s, t}(P, \mathbf{Z} / 2)=0$ for $t-s>\left(2^{i}-2\right) s$ $+m$, for $P$ any $A_{i-1}$-module having $P_{r}=0$ for $r>m$. Consider any $A_{i}$-module $M$ satisfying the hypothesis of the theorem. Then there is a KSS:

$$
\operatorname{Ext}_{A_{i-1}}^{s-\sigma, t}\left(N_{\sigma}(i) \otimes M, \mathbf{Z} / 2\right) \rightarrow \operatorname{Ext}_{A_{i}}^{s, t}(M, \mathbf{Z} / 2) .
$$

The top class of $N_{\sigma} \otimes M$ is in dimension $\leqslant\left(2^{i+1}-1\right) \sigma+m$, by our definition of $N_{\sigma}$. Thus,

$$
\begin{array}{r}
\operatorname{Ext}_{A_{i-1}}^{s-\sigma, t}\left(N_{\boldsymbol{\sigma}}(i) \otimes M, \mathbf{Z} / 2\right)=0 \text { for } t-s+\sigma>\left(2^{i}-2\right)(s-\sigma) \\
+\left(2^{i+1}-2\right) \sigma+m
\end{array}
$$

i.e.

$$
t-s>\left(2^{i}-2\right) s+2^{i} \sigma+m .
$$

Since $0 \leqslant \sigma \leqslant s$, we have $\operatorname{Ext}_{A_{i}}^{s, t}(M, \mathbf{Z} / 2)=0$ for $t-s>\left(2^{i+1}-2\right) s+m$.

3. Some periodicity elements. In this section, we use the machinery developed in $\S 2$ to construct certain periodicity elements in $\operatorname{Ext}_{A_{i}}(\mathbf{Z} / 2, \mathbf{Z} / 2)$. Our first main result is

TheOREM A. For $i$ any positive integer, there exists a unique nonzero divisor $w_{i} \in \operatorname{Ext}_{A_{i}}^{2^{i+1}, 2^{i+1}\left(2^{i+1}-1\right)}(\mathbf{Z} / 2, \mathbf{Z} / 2)$ such that $w_{i}$ restricts nontrivially to $\operatorname{Ext}_{E\left[Q_{i}\right]}(\mathbf{Z} / 2, \mathbf{Z} / 2)$, corresponding to the class $v_{i}^{2^{i+1}} \in \pi_{*}(\mathrm{BP})$.

We hereafter denote $w_{i}$ as $v_{i}^{2^{i+1}} \in \operatorname{Ext}_{A_{i}}(\mathbf{Z} / 2, \mathbf{Z} / 2)$.

Proof. Consider the module $R_{\sigma}(i)$ defined in $\S 2$, with $\sigma=2^{i+1}$. The top class in $R_{\sigma}(i)$ is $\zeta_{i+1}\left|\zeta_{i+1}\right| \cdots \mid \zeta_{i+1}=\left(\zeta_{i+1}\right)^{\sigma}$. Define maps $\mathbf{Z} / 2 \stackrel{g}{\rightarrow} R_{\sigma}(i) \stackrel{h}{\rightarrow} \mathbf{Z} / 2$ by $g(1)$ $=\left(\zeta_{i+1}\right)^{\sigma}, h\left[\left(\zeta_{i+1}\right)^{\sigma}\right]=1$, both 0 otherwise. $\mathrm{Sq}^{2^{i+1}}=\mathrm{Sq}^{\sigma}$ acts nontrivially on the class $\left(\zeta_{i+1}\right)^{\sigma}$, but $A_{i}$ acts trivially on it since $A_{i}$ acts trivially on any $2^{i+1}$ st power. So $h$ and $g$ are $A_{i}$-module maps that split the class $\left(\zeta_{i+1}\right)^{\sigma}$ off from $R_{\sigma}(i)$. We can tensor with $E(i)$ to get $E(i) \stackrel{\bar{g}}{\rightarrow} E(i) \otimes R_{\sigma}(i) \stackrel{\bar{h}}{\rightarrow} E(i)$. Dualizing, we get

$$
A_{i} \otimes_{A_{i-1}} \mathbf{Z} / 2 \stackrel{\hat{h}}{\rightarrow} A_{i} \otimes_{A_{i-1}} N_{\sigma}(i) \stackrel{\hat{g}}{\rightarrow} A_{i} \otimes_{A_{i-1}} \mathbf{Z} / 2 .
$$

This extends to a splitting of complexes:

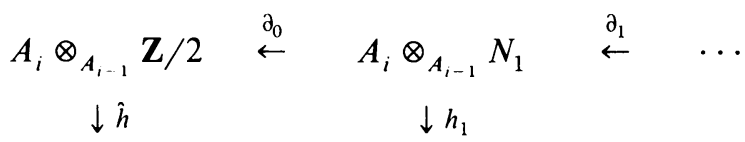

$$
\begin{aligned}
& \ldots \stackrel{\partial_{\sigma-2}}{\leftarrow} A_{i} \otimes_{A_{i-1}} N_{\sigma-1} \stackrel{\partial_{\sigma-1}}{\leftarrow} \quad A_{i} \otimes_{A_{i-1}} N_{\sigma} \stackrel{\partial_{\sigma}}{\leftarrow} \quad A_{i} \otimes_{A_{i-1}} N_{\sigma+1} \stackrel{\partial_{\sigma+1}}{\leftarrow} \quad \ldots \\
& \downarrow \hat{g} \quad \downarrow g_{1} \\
& A_{i} \otimes_{A_{i}, 1} \mathrm{Z} / 2 \quad \stackrel{\partial_{0}}{\leftarrow} \quad A_{i} \otimes_{A_{i-1}} N_{1} \stackrel{\partial_{1}}{\leftarrow} \quad \ldots
\end{aligned}
$$


Here, $h_{i}(x \otimes y)=h(x) \otimes\left[\left(\zeta_{i+1}\right)^{\sigma^{*}} y\right]$ and

$$
g_{i}(x \otimes y)= \begin{cases}x \otimes r & \text { if } y=\left(\zeta_{i+1}\right)^{\sigma^{*}} \cdot r, \\ 0 & \text { if }\left(\zeta_{i+1}\right)^{\sigma^{*}}+y .\end{cases}
$$

Also, it is understood that $N_{0}(i) \cong \mathbf{Z} / 2$. Recall that the $\operatorname{KSS}_{\text {for }} \operatorname{Ext}_{A_{i}}(\mathbf{Z} / 2, \mathbf{Z} / 2)$ is obtained by applying $\operatorname{Ext}_{A_{i}}^{s-\sigma, t}(-, \mathbf{Z} / 2)$ to the $K R_{i}$ complex. Our diagram (3.2) is a splitting of that complex. In fact, let $g^{\prime}$ denote the composition

$$
A_{i} \otimes_{A_{i-1}} N_{\sigma} \stackrel{\hat{g}}{\rightarrow} A_{i} \otimes_{A_{i, 1}} \mathbf{Z} / 2 \stackrel{\text { augment }}{\rightarrow} \mathbf{Z} / 2
$$

Then

$$
g^{\prime} \in \operatorname{Hom}_{A_{i}}^{t}\left(A_{i} \otimes_{A_{t-1}} N_{\sigma}, \mathbf{Z} / 2\right)=\operatorname{Ext}_{A_{i}}^{0, t}\left(A_{i} \otimes_{A_{i},} N_{\sigma}, \mathbf{Z} / 2\right),
$$

where $t=2^{i+1}\left(2^{i+1}-1\right)$. So $g^{\prime}$ arises in the $E_{1}$-term of the KSS: $g^{\prime} \in E_{1}^{\sigma, 2^{i+1}, t}$. To see that the class given by $g^{\prime},\left(g^{\prime}\right)$, is a cycle in the KSS, note that $\left(e \otimes\left(\zeta_{i+1}\right)^{\sigma^{*}}\right)$ is in the image of the map

$$
\operatorname{Ext}_{A_{i}}\left(M_{\sigma+1}, \mathbf{Z} / 2\right) \rightarrow \operatorname{Ext}_{A_{i}}\left(A_{i} \otimes_{A_{i-1}} N_{\sigma}, \mathbf{Z} / 2\right) \text { (diagram 2.6). }
$$

Thus $\left(g^{\prime}\right)$ is a cycle by standard homological algebra arguments. Further, $\left(g^{\prime}\right)$ is never a boundary since $d_{r} x=\left(g^{\prime}\right)$ implies that $x$ lies in a subquotient of $\operatorname{Ext}_{\boldsymbol{A}_{i-1}}^{r-1, t}\left(N_{\sigma-r}, \mathbf{Z} / 2\right)$, which is zero for $r<2^{i+1}$ by the ledge theorem. Thus, $\left(g^{\prime}\right)$ projects to a nontrivial class $w_{i} \in \operatorname{Ext}_{A_{i}}^{2^{i+1} \cdot t}(\mathbf{Z} / 2, \mathbf{Z} / 2)$. This class is a nonzero divisor in $\operatorname{Ext}_{A_{i}}(\mathbf{Z} / 2, \mathbf{Z} / 2)$ because it is obtained from a full splitting of complexes. More precisely, the Yoneda product $w_{i} a \neq 0$ whenever $a \neq 0$ in $\operatorname{Ext}_{A_{i}}(\mathbf{Z} / 2, \mathbf{Z} / 2)$.

We identify this class $w_{i}$ in the setting of $\pi_{*}(\mathrm{BP})=\mathbf{Z}_{(2)}\left[v_{1}, v_{2}, \ldots\right]$. Consider the Baas-Sullivan spectrum $\mathrm{BP}\langle i\rangle[3]$, where $\pi_{*}(\mathrm{BP}\langle i\rangle)=\mathbf{Z}_{(2)}\left[v_{1}, v_{2}, \ldots, v_{i}\right]$. The $\bmod 2$ cohomology is given by $H^{*} \mathrm{BP}\langle i\rangle=A \otimes_{E\left(Q_{0}, Q_{1}, \ldots, Q_{1}\right)} \mathbf{Z} / 2$, where $Q_{j}$ denotes the Milnor generator, and the clASS connecting the cohomology and the homotopy collapses:

$$
\begin{aligned}
E_{2}^{* *} & =\operatorname{Ext}_{A}^{* *}\left(H^{*} \mathrm{BP}\langle i\rangle, \mathbf{Z} / 2\right)=\operatorname{Ext}_{A}^{* *}\left(A \otimes_{E\left(Q_{0}, Q_{1}, \ldots, Q_{,}\right)} \mathbf{Z} / 2, \mathbf{Z} / 2\right) \\
& \approx \operatorname{Ext}_{E\left(Q_{0}, Q_{1} \ldots, Q_{i}\right)}^{* *}(\mathbf{Z} / 2, \mathbf{Z} / 2)
\end{aligned}
$$

by change of rings

$$
\cong \mathbf{Z} / 2\left[h_{0}, v_{1}, v_{2}, \ldots, v_{i}\right] \Rightarrow \pi_{*}(\mathrm{BP}\langle i\rangle)=\mathbf{Z}_{(2)}\left[v_{1}, v_{2}, \ldots, v_{i}\right],
$$

since $h_{0}$ corresponds to multiplication by 2 . We can think of $H^{*} \mathrm{BP}\langle i\rangle$ as the extended $A$-module $A \otimes_{A} A_{i} \otimes_{E\left(Q_{0}, Q_{1}, \ldots, Q_{i}\right)} \mathbf{Z} / 2$ since $E\left(Q_{0}, Q_{1}, \ldots, Q_{i}\right)$ is a subalgebra of $A_{i}$. Thus,

$$
\operatorname{Ext}_{A}\left(H^{*} \mathrm{BP}\langle i\rangle, \mathbf{Z} / 2\right)=\operatorname{Ext}_{A_{i}}\left(A_{i} \otimes_{E\left(Q_{0}, Q_{1}, \ldots, Q_{,}\right)} \mathbf{Z} / 2, \mathbf{Z} / 2\right) .
$$

Note that $A_{i} \otimes_{E\left(Q_{0}, Q_{1}, \ldots, Q_{i}\right)} \mathbf{Z} / 2 \cong \mathscr{D} A_{i-1}$, the double of $A_{i-1}$, as an $A_{i}$-module and as an algebra. By this we mean that $A_{i} \otimes_{E\left(Q_{0}, Q_{1}, \ldots, Q_{1}\right)} \mathbf{Z} / 2$ is isomorphic to the image of $A_{i-1}$ under the doubling homomorphism in $A$ [16]. Thus we have the clASS for $\mathrm{BP}\langle i\rangle$ :

$$
E_{2}^{* *}(\mathrm{BP}\langle i\rangle) \cong \operatorname{Ext}_{A_{i}}^{* *}\left(\mathscr{D} A_{i-1}, \mathbf{Z} / 2\right) \rightarrow \pi_{*}(\mathrm{BP}\langle i\rangle)=\mathbf{Z}_{(2)}\left[v_{1}, v_{2}, \ldots, v_{i}\right]
$$


Hence, there is a class at $s=2^{i+1}, t=2^{i+1}\left(2^{i+1}-1\right)$ in $\operatorname{Ext}_{A_{i}}\left(\mathscr{D} A_{i-1}, \mathbf{Z} / 2\right)$ representing $v_{i}^{i+1} \in \pi_{*}(\mathrm{BP}\langle i\rangle)$. The augmentation $\mathscr{D} A_{i-1} \stackrel{j}{\rightarrow} \mathrm{Z} / 2$ induces a map $\operatorname{Ext}_{A_{i}}^{* *}(\mathbf{Z} / 2, \mathbf{Z} / 2) \stackrel{j^{*}}{\rightarrow} \operatorname{Ext}_{\boldsymbol{A}_{i}}^{* *}\left(\mathscr{D} A_{i-1}, \mathbf{Z} / 2\right)$. Then $j^{*} w_{i}=\left(v_{i}^{2^{i+1}}\right)$. This follows since the May spectral sequence for $\operatorname{Ext}_{A_{i}}(\mathbf{Z} / 2, \mathbf{Z} / 2)$ shows that $w_{i}$ is the only nontrivial class present in the bigrading $s, t=2^{i+1}, 2^{i+1}\left(2^{i+1}-1\right)$. Also $\left(v_{i}^{2+1}\right)$ is the unique class in $\operatorname{Ext}_{A_{i}}\left(\mathscr{D} A_{i-1}, \mathbf{Z} / 2\right)$ at that bigrading. Both have the same May SS representative: $b_{0, i+1}^{2^{\prime}}$. Since both classes are nontrivial, we have established that $j^{*}\left(w_{i}\right)=$ $\left(v_{i}^{2^{i+1}}\right)$. This completes the proof of Theorem A.

We now use the classes $v_{i}^{2^{i+1}} \in \operatorname{Ext}_{A_{i}}(\mathbf{Z} / 2, \mathbf{Z} / 2)$ to produce periodicity operators in the cohomology of the Steenrod algebra. J. F. Adams was the first to note the existence of periodic phenomena in the $E_{2}$-term of the clASS [1]. In that paper, he constructed an element corresponding to $v_{1}^{4}$ in $\operatorname{Ext}_{A_{1}}(\mathbf{Z} / 2, \mathbf{Z} / 2)$. Further, he showed that $v_{1}^{2^{k}} \in \operatorname{Ext}_{A_{k}}(\mathbf{Z} / 2, \mathbf{Z} / 2)$ for $k \geqslant 2$. Using this, a periodicity operator is defined:

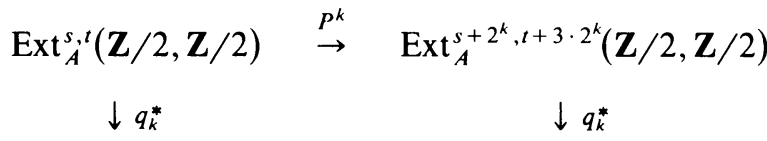

$$
\operatorname{Ext}_{\boldsymbol{A}_{k}}^{s, t}(\mathbf{Z} / 2, \mathbf{Z} / 2) \quad \stackrel{v_{1}^{2^{k}}}{\rightarrow} \quad \operatorname{Ext}_{A_{k}}^{s+2^{k}, t+3 \cdot 2^{k}}(\mathbf{Z} / 2, \mathbf{Z} / 2)
$$

$P^{k} x$ is defined whenever $q_{k}^{*}(x) \neq 0$, with $P^{k} x$ being the coset pulled back from $v_{1}^{2^{k}} \cdot q_{k}^{*}(x)$. This can be expressed as a Massey product: $P^{1} x=\left\langle h_{3}, h_{0}^{4}, x\right\rangle$, iterated to give $P^{k}$ for $k>1$. This operator is an isomorphism in $\operatorname{Ext}_{A}^{s, t}(\mathbf{Z} / 2, \mathbf{Z} / 2)$ in a neighborhood of the line of slope $\frac{1}{2}$. An element $x \in \operatorname{Ext}(\mathbf{Z} / 2, \mathbf{Z} / 2)$ is periodic under the Adams operator if $P^{k} x \neq 0$ for $k \geqslant 1$.

Our goal is now to define the notion of $v_{i}$-periodicity in $\operatorname{Ext}_{A}(\mathbf{Z} / 2, \mathbf{Z} / 2)$ using the elements $v_{i}^{2^{i+1}} \in \operatorname{Ext}_{A_{i}}(\mathbf{Z} / 2, \mathbf{Z} / 2)$ constructed in the proof of Theorem A. To begin, we need a result along the lines of Adams' proof that $v_{1}^{2^{k}}$ lives in $\operatorname{Ext}_{A_{1}}, \operatorname{Ext}_{A_{2}}, \ldots$, up to $\operatorname{Ext}_{A_{k}}, k \geqslant 2$.

THEOREM B. For $k$ any positive integer, there exists a sequence of positive integers $N_{1}, N_{2}, \ldots, N_{k}$ such that

$$
\mathbf{Z} / 2\left[h_{0}, v_{1}^{\left(4 N_{1}\right)}, v_{2}^{\left(8 N_{2}\right)}, \ldots, v_{i}^{\left(2^{i+1} N_{i}\right)}, \ldots, v_{k}^{\left(2^{k+1} N_{k}\right)}\right] \subset \operatorname{Ext}_{A_{k}}(\mathbf{Z} / 2, \mathbf{Z} / 2) .
$$

Note that $N_{i}$ also depends on the value of $k$. Also note that $N_{k}$ can be chosen to be 1 by Theorem $A$.

PROOF. The following result is proved in [12] by Lin. Another proof was presented later by Wilkerson in [18].

THEOREM (3.4). If $B$ is a Hopf subalgebra of a finite, graded, connected, cocommutative Hopf algebra A, then the restriction map

$$
i^{*}: \operatorname{Ext}_{A}(\mathbf{Z} / 2, \mathbf{Z} / 2) \rightarrow \operatorname{Ext}_{B}(\mathbf{Z} / 2, \mathbf{Z} / 2) / \text { nilpotents }
$$

is nonzero in infinitely many positive degrees. 
Wilkerson's proof uses the natural action of the Steenrod algebra in the LyndonHochschild-Serre spectral sequence, together with the observation that the cohomology of a finite, connected, cocommutative Hopf algebra is Noetherian. To apply this theorem to our case, we note that there are exterior subalgebras of $A_{i}, E\left(Q_{0}\right)$, $E\left(Q_{1}\right), E\left(Q_{0}, Q_{1}\right), \ldots, E\left(Q_{0}, Q_{1}, \ldots, Q_{i}\right)$. Apply Lin's theorem with $A=A_{k}$ and $B=E\left(Q_{i}\right)$. Now $\operatorname{Ext}_{E\left(Q_{0}, Q_{1}, \ldots, Q_{i}\right)}(\mathbf{Z} / 2, \mathbf{Z} / 2) \cong \mathbf{Z} / 2\left[h_{0}, v_{1}, \ldots, v_{i}\right]$. Define the class $v_{i}^{N} \in \operatorname{Ext}_{A_{k}}(\mathbf{Z} / 2, \mathbf{Z} / 2)$ to be the coset of elements that map to the class $v_{i}^{N} \in$ $\operatorname{Ext}_{E\left(Q_{0}, Q_{1}, \ldots, Q_{i}\right)}(\mathbf{Z} / 2, \mathbf{Z} / 2) \cong \mathbf{Z} / 2\left[h_{0}, v_{1}, \ldots, v_{i}\right]$. This must be nontrivial for some sufficiently large $N$, completing the proof of Theorem B.

ReMARKs. (1) One should note that $h_{0}$ lives in all $\operatorname{Ext}_{A_{k}}(\mathbf{Z} / 2, \mathbf{Z} / 2)$ 's.

(2) While $v_{i}^{2^{i+1}}$ is an element in $\operatorname{Ext}_{A_{i}}(\mathbf{Z} / 2, \mathbf{Z} / 2), \quad v_{i}^{2^{i+1} N_{i}}$ is a coset in $\operatorname{Ext}_{A_{k}}(\mathbf{Z} / 2, \mathbf{Z} / 2)$ for $k>i$.

(3) The natural projections

$$
p_{k-1}: \operatorname{Ext}_{A_{k}}(\mathbf{Z} / 2, \mathbf{Z} / 2) \rightarrow \operatorname{Ext}_{A_{k-1}}(\mathbf{Z} / 2, \mathbf{Z} / 2)
$$

satisfy

$$
p_{k-1}\left(v_{i}^{N}\right) \subset v_{i}^{N} \in \operatorname{Ext}_{A_{i, 1}}(\mathbf{Z} / 2, \mathbf{Z} / 2) .
$$

This follows since the restriction maps and projections are induced from:

$$
\begin{array}{ccc}
E\left(Q_{1}, Q_{2}, \ldots, Q_{i-1}\right) & \hookrightarrow & E\left(Q_{1}, Q_{2}, \ldots, Q_{i}\right) \\
\downarrow & & \downarrow \\
A_{i-1} & \hookrightarrow & A_{i}
\end{array}
$$

(4) Given $k>i$, the smallest power of $v_{i}^{i+1}$ that could be present in $\operatorname{Ext}_{A_{k}}(\mathbf{Z} / 2, \mathbf{Z} / 2)$ is $2^{k-i+1}$. If any smaller power were present, then it would be in the image of $\operatorname{Ext}_{A}(\mathbf{Z} / 2, \mathbf{Z} / 2)$ by the Adams approximation theorem [1]. This is impossible, since all powers of $v_{i}$ must support an $h_{0}$-tower, contradicting the Adams edge theorem [1].

We can summarize these results in the following tower.

$$
\begin{array}{cccc}
\operatorname{Ext}_{A}(\mathbf{Z} / 2, \mathbf{Z} / 2)=\lim _{k} \operatorname{Ext}_{A_{k}}(\mathbf{Z} / 2, \mathbf{Z} / 2) & \downarrow \\
& & \vdots \\
& & & \\
& & p_{k} \\
\left(v_{i}^{2^{i+1}}\right)^{M_{k}} & \subset & \operatorname{Ext}_{A_{k}}(\mathbf{Z} / 2, \mathbf{Z} / 2) \\
& & \downarrow p_{k-1} \\
\left(v_{i}^{2^{i+1}}\right)^{M_{k-1}} & \subset & \operatorname{Ext}_{A_{k-1}}(\mathbf{Z} / 2, \mathbf{Z} / 2) \\
& & \downarrow p_{k-2} \\
& & \vdots \\
& & \downarrow p_{i} \\
v_{i}^{2^{i+1}} & & \in & \operatorname{Ext}_{A_{i}}(\mathbf{Z} / 2, \mathbf{Z} / 2)
\end{array}
$$

In (3.5), $M_{k} \geqslant M_{k-1}$ and $\lim M_{k}=\infty$. 
We know, then, that for $k \geqslant i$, there is a polynomial algebra on $v_{i}^{2^{i+1} N_{i}}$ present in $\operatorname{Ext}_{A_{k}}(\mathbf{Z} / 2, \mathbf{Z} / 2)$. It is reasonable to ask what is the lowest power of $v_{i}^{2^{i+1}}$ that can live in $\operatorname{Ext}_{A_{k}}(\mathbf{Z} / 2, \mathbf{Z} / 2)$. There is substantial evidence that the answer is this.

ConJeCTURE (3.6). $v_{i}^{2^{i+1+m}}$ is present in $\operatorname{Ext}_{A_{k}}(\mathbf{Z} / 2, \mathbf{Z} / 2)$ if and only if $i \leqslant i \leqslant 2 i$ $+m$.

To define the notion of $v_{i}$-periodicity and $v_{i}$-torsion in $\operatorname{Ext}_{A}(\mathbf{Z} / 2, \mathbf{Z} / 2)$, we will localize each $\operatorname{Ext}_{A_{k}}(\mathbf{Z} / 2, \mathbf{Z} / 2)$ with respect to $v_{i}$ for each $k \geqslant i$. Since these localizations commute with the natural projections (Remark (3)) they must commute with the inverse limit. To be clear about what we mean by localization with respect to the coset $v_{i}^{N}$, let $N$ be such that $v_{i}^{N}$ is the smallest power of $v_{i}^{2^{i+1}}$ present in $\operatorname{Ext}_{A_{k}}(\mathbf{Z} / 2, \mathbf{Z} / 2)$. Let $\left(a_{1}, a_{2}, \ldots, a_{m}\right)$ be the full coset $v_{i}^{N}$. It is finite since $\operatorname{Ext}_{A_{k}} t_{A^{\prime}}(\mathbf{Z} / 2, \mathbf{Z} / 2)$ is finite for any $s, t$. We can then form the element $a=a_{1} a_{2} \cdots a_{m}$, which will be a uniquely determined element in the coset $\left(v_{i}^{N}\right)^{m}$. Then $\operatorname{Ext}_{A_{k}}(\mathbf{Z} / 2, \mathbf{Z} / 2)\left(\left(v_{i}\right)^{-1}\right)$ is defined as the direct limit of the sequence

$$
\operatorname{Ext}_{A_{k}} \stackrel{\cdot a}{\rightarrow} \Sigma^{-N m\left(2^{i+1}-2\right)} \operatorname{Ext}_{A_{k}} \stackrel{\cdot a}{\rightarrow} \Sigma^{-2 N m\left(2^{i+1}-2\right)} \operatorname{Ext}_{A_{k}} \stackrel{\cdot a}{\rightarrow} \cdots .
$$

With this in mind, we use $\operatorname{Ext}_{A_{k}}(\mathbf{Z} / 2, \mathbf{Z} / 2)\left(\left(v_{i}\right)^{-1}\right)$ to denote localization with respect to this uniquely determined power of $v_{i}^{2^{i+1}}$ in $\operatorname{Ext}_{A_{k}}(\mathbf{Z} / 2, \mathbf{Z} / 2)$. Since $p_{k-1}\left(v_{i}^{N}\right) \subset v_{i}^{N} " \in " \operatorname{Ext}_{A_{k-1}}(\mathbf{Z} / 2, \mathbf{Z} / 2)$, these localizations fit together into the following tower:

$$
\begin{aligned}
& \vdots \\
& \operatorname{Ext}_{A_{k}}(\mathbf{Z} / 2, \mathbf{Z} / 2) \quad \underset{\text { invert } v_{i}}{\stackrel{f_{i}^{k}}{\rightarrow}} \quad \operatorname{Ext}_{A_{k}}(\mathbf{Z} / 2, \mathbf{Z} / 2)\left(v_{i}^{-1}\right)
\end{aligned}
$$

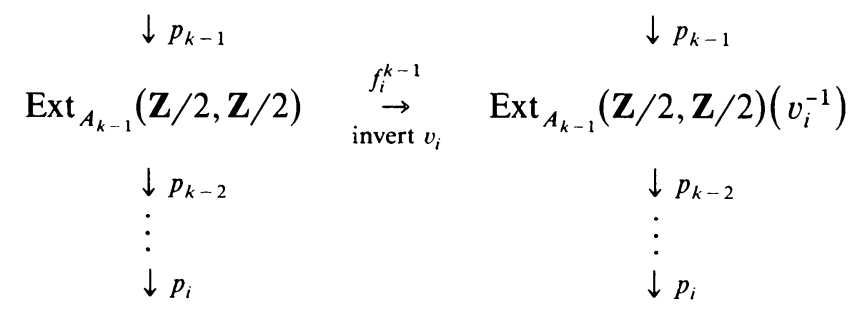

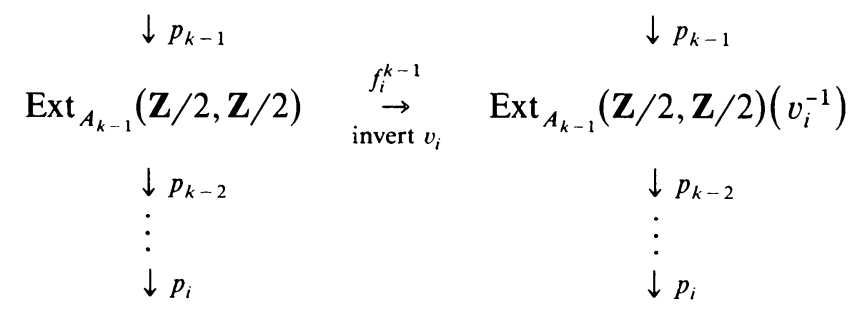

$$
\begin{aligned}
& \operatorname{Ext}_{A_{i}}(\mathbf{Z} / 2, \mathbf{Z} / 2) \quad \underset{\text { invert } v_{i}}{\stackrel{f_{i}^{i}}{\rightarrow}} \operatorname{Ext}_{A_{i}}(\mathbf{Z} / 2, \mathbf{Z} / 2)\left(v_{i}^{-1}\right)
\end{aligned}
$$

Since the tower commutes, we can form the inverse limit: let

$$
V_{i}^{s, t}=\underset{k}{\lim _{\leftarrow}}\left[\operatorname{Ext}_{A_{k}}^{s, t}(\mathbf{Z} / 2, \mathbf{Z} / 2)\left(v_{i}^{-1}\right)\right]
$$

Then we have a map $f_{i}$ given by

$$
\begin{aligned}
& \operatorname{Ext}_{A}^{s, t}(\mathbf{Z} / 2, \mathbf{Z} / 2) \quad \stackrel{f_{i}}{\rightarrow} \quad V_{i}^{s, t} \\
& \|\quad\| \text { def } \\
& \lim _{k} \operatorname{Ext}_{A_{k}}^{s, t}(\mathbf{Z} / 2, \mathbf{Z} / 2) \stackrel{\lim _{\leftarrow} f_{i}^{k}}{\rightarrow} \lim _{k}\left[\operatorname{Ext}_{A_{k}}^{s, t}(\mathbf{Z} / 2, \mathbf{Z} / 2)\left(v_{i}^{-1}\right)\right] .
\end{aligned}
$$


Definition (3.8). An element $x \in \operatorname{Ext}_{A}(\mathbf{Z} / 2, \mathbf{Z} / 2)$ is $v_{i}$-periodic if $f_{i}(x) \neq 0$ and $v_{i}$-torsion otherwise.

Equivalently, $x \in \operatorname{Ext}_{A}(\mathbf{Z} / 2, \mathbf{Z} / 2)$ is $v_{i}$-periodic if there exists an integer $M \geqslant 0$ such that $q_{k}^{*}(x)\left(v_{i}^{N_{i}}\right)^{s} \neq 0$ for all $s>0$, all $k \geqslant M$. Here $q_{k}^{*}: \operatorname{Ext}_{A}(\mathbf{Z} / 2, \mathbf{Z} / 2) \rightarrow$ $\operatorname{Ext}_{A_{k}}(\mathbf{Z} / 2, \mathbf{Z} / 2)$ denotes the natural projection and $v_{i}^{N_{1}}$ “ $\in$ " $\operatorname{Ext}_{A_{k}}(\mathbf{Z} / 2, \mathbf{Z} / 2)$ is the smallest nonzero power of $v_{i}^{2^{i+1}}$ present there. $x \in \operatorname{Ext}_{A}(\mathbf{Z} / 2, \mathbf{Z} / 2)$ is $v_{n}$-torsion if there exists some $M \geqslant 0$ such that for all $k \geqslant M$ there is an $s>0$ with $q_{k}^{*}(x)\left(v_{i}^{N_{i}}\right)^{s}$ $=0$ in $\operatorname{Ext}_{A_{k}}(\mathbf{Z} / 2, \mathbf{Z} / 2)$.

4. Operations on $\operatorname{Ext}_{A_{k}}(\mathbf{Z} / 2, \mathbf{Z} / 2)$. In this section, we construct certain families of operations

$$
r_{j}: \operatorname{Ext}_{A_{k}}(\mathbf{Z} / 2, \mathbf{Z} / 2) \rightarrow \operatorname{Ext}_{A_{k}{ }_{1}}\left(\Sigma^{j 2^{k+1}} \mathbf{Z} / 2, \mathbf{Z} / 2\right)
$$

for $k \geqslant 1$ which are used to prove Theorem $C$. These operations are constructed using the first stage of the resolution constructed in [11], and are related to the Quillen operations in $\mathrm{BP}_{*}$. We show how these operations act on the periodicity elements $v_{i}^{N} \in \operatorname{Ext}_{A_{k}}(\mathbf{Z} / 2, \mathbf{Z} / 2)$ constructed earlier.

The operations are induced by a map given by Theorem 5 of [11],

$$
\bar{\phi}_{k}: \bigoplus_{m \geqslant 0} \Sigma^{m 2^{k+1}} A / / A_{k-1} \rightarrow A / / A_{k}
$$

defined by $\bar{\phi}_{k}\left(i_{m}\right)=\chi \mathrm{Sq}^{m 2^{k+1}}$, where $i_{m}$ denotes the generator of the $m$ th summand. The dual of this map is easily described. Recalling that

$$
\left(A / / A_{n}\right)^{*} \cong \mathbf{Z} / 2\left[\zeta_{1}^{2^{n+1}}, \zeta_{2}^{2^{n}}, \ldots, \zeta_{n+1}^{2}, \zeta_{n+2}, \zeta_{n+3}, \ldots\right],
$$

there is an isomorphism

$$
\bigoplus_{m \geqslant 0} \Sigma^{m 2^{k+1}}\left(A / / A_{k-1}\right)^{*} \cong \mathbf{Z} / 2\left[t^{2^{k+1}}, \zeta_{1}^{2^{k}}, \zeta_{2}^{2^{k-1}}, \ldots, \zeta_{k}^{2}, \zeta_{k+1}, \ldots\right]
$$

where $t$ is a placeholder with $|t|=1$ and $t \mathrm{Sq}=0$.

LeMma (4.1). The dual of $\bar{\phi}_{k}$ is given by

$$
\phi_{k}: \mathbf{Z} / 2\left[\zeta_{1}^{2^{k+1}}, \zeta_{2}^{2^{k}}, \ldots, \zeta_{k+1}^{2}, \ldots\right] \rightarrow \mathbf{Z} / 2\left[t^{2^{k+1}}, \zeta_{1}^{2^{k}}, \zeta_{2}^{2^{k}}, \ldots, \zeta_{k}^{2}, \zeta_{k+1}, \ldots\right] .
$$

Here $\phi_{k}$ is defined on the generators by

$$
\phi_{k}\left(\zeta_{j}^{2^{n}}\right)=\zeta_{j}^{2^{n}}+\zeta_{j-1}^{2^{n}} t^{2^{n+j-1}} \quad \text { where } n= \begin{cases}k+2-j & \text { if } j<k+2 \\ 0 & \text { if } j \geqslant k+2\end{cases}
$$

Extending $\phi_{k}$ over all of $\left(A / / A_{k}\right)^{*}$ by multiplicativity completes the definition.

Proof. The definition of $\bar{\phi}_{k}$ and an exercise in duality show that $\phi_{k}$ can be computed as follows: let $A / / A_{k}^{*} \stackrel{\psi}{\rightarrow} A^{*} \otimes A / / A_{k}^{*}$ denote the coaction of the dual of the Steenrod algebra on $A / / A_{k}^{*}$. Then for $R$ any sequence of nonnegative integers, there exist integers $\varepsilon_{m}$ and squences $R_{m}, I_{t}$ and $J_{t}$ such that

$$
\psi\left(\zeta^{R}\right)=\sum_{m} \varepsilon_{m} \zeta_{1}^{m 2^{k+1}} \otimes \zeta^{R_{m}}+\sum_{t} \zeta^{I_{t}} \otimes \zeta^{J_{t}}
$$


where $\varepsilon_{m}=0$ or 1 and $i_{1}=0$ in $I_{t}$. Then $\left(\bar{\phi}_{k}\right)^{*}\left(\zeta^{R}\right)=\oplus_{m} \varepsilon_{m} \zeta^{R_{m}} m 2^{k+1}$. This gives precisely the definition of $\phi_{k}$.

Note that the map $\phi_{k}$ also respects the right $A$-module structure involved since $\left(\zeta_{k}\right) \mathrm{Sq}=\zeta_{k}+\zeta_{k-1}$. Thus, $\phi_{k}$ induces a map in $\operatorname{Ext}_{A}(\mathbf{Z} / 2,-)$ :

$$
\begin{aligned}
& \operatorname{Ext}_{A}\left(\mathbf{Z} / 2,\left(A / / A_{k}\right)^{*}\right) \stackrel{\phi_{k^{*}}}{\rightarrow} \operatorname{Ext}_{A}\left(\mathbf{Z} / 2, \bigoplus_{m \geqslant 0} \sum^{m 2^{k+1}}\left(A / / A_{k-1}\right)^{*}\right) \\
& \cong \downarrow \begin{array}{l}
\text { Change } \\
\text { of } \\
\text { rings }
\end{array} \quad \cong \downarrow \begin{array}{l}
\text { of } \\
\text { rings }
\end{array} \\
& \operatorname{Ext}_{A_{k}}(\mathbf{Z} / 2, \mathbf{Z} / 2) \quad \stackrel{r}{\rightarrow} \quad \bigoplus_{m \geqslant 0} \operatorname{Ext}_{A_{k-1}}\left(\Sigma^{m 2^{k+1}} \mathbf{Z} / 2, \mathbf{Z} / 2\right) .
\end{aligned}
$$

Here all four objects are rings, with the ring structures on the top row inherited from those on $\left(A / / A_{k}\right)^{*}$ and $\oplus_{m \geqslant 0} \Sigma^{m 2^{k+1}}\left(A / / A_{k-1}\right)^{*}$. The bottom row has ring structures given by Yoneda product. Now $\bar{\phi}_{k^{*}}$ is a ring homomorphism since $\bar{\phi}_{k}$ is, and the change of rings isomorphism respects these structures, so that the map $r$ is also a ring homomorphism.

We break $r$ into its components $r=\oplus_{m \geqslant 0} r_{m}$ where

$$
r_{m}: \operatorname{Ext}_{A_{k}}(\mathbf{Z} / 2, \mathbf{Z} / 2) \rightarrow \operatorname{Ext}_{A_{k-1}}\left(\Sigma^{m 2^{k+1}} \mathbf{Z} / 2, \mathbf{Z} / 2\right) .
$$

Then the ring structure of $r$ is a Cartan formula:

$$
r_{m}(x y)=\bigoplus_{j=0}^{m} r_{j}(x) r_{m-j}(y) .
$$

Notice also

$$
r_{0}(x)=p_{k-1}(x)
$$

where $p_{k-1}: \operatorname{Ext}_{A_{k}}(\mathbf{Z} / 2, \mathbf{Z} / 2) \rightarrow \operatorname{Ext}_{A_{k-1}}(\mathbf{Z} / 2, \mathbf{Z} / 2)$ is induced from the inclusion. Finally, if $x \in \operatorname{Ext}_{A}(\mathbf{Z} / 2, \mathbf{Z} / 2)$ and $x^{\prime}=q_{k}(x) \in \operatorname{Ext}_{A_{k}}(\mathbf{Z} / 2, \mathbf{Z} / 2)$, then $r_{m}\left(x^{\prime}\right)=$ $p_{k-1}\left(x^{\prime}\right)$ if $m=0$, zero otherwise. This follows since the map $\phi_{k}$ is a map of $A$-modules, so that the map induced in $\operatorname{Ext}_{A}(-, \mathbf{Z} / 2)$ must respect Yoneda products with classes from $\operatorname{Ext}_{A}(\mathbf{Z} / 2, \mathbf{Z} / 2)$.

We now consider the action of these operations on the periodicity classes $v_{j}^{2^{m}} \subset \operatorname{Ext}_{A_{n}}(\mathbf{Z} / 2, \mathbf{Z} / 2)$. To do this, we consider $\operatorname{Ext}_{A}\left(A / / E_{n}, \mathbf{Z} / 2\right) \cong$ $\operatorname{Ext}_{E_{n}}(\mathbf{Z} / 2, \mathbf{Z} / 2)$, where $E_{n}$ denotes the exterior algebra $E\left(Q_{0}, \ldots, Q_{n}\right) \subset A_{n}$. Recall that $\operatorname{Ext}_{E_{n}}(\mathbf{Z} / 2, \mathbf{Z} / 2)=\mathbf{Z} / 2\left[v_{0}, v_{1}, \ldots, v_{n}\right]$, and that there is a natural restriction map $j_{n}: \operatorname{Ext}_{A_{n}} \rightarrow \operatorname{Ext}_{E_{n}}$. Let $K_{n}$ denote the kernel of $j_{n}$. Then the operations constructed above act on these periodicity classes in the following manner.

TheOREM (4.5). For the classes $v_{j}^{2^{m}} \in \operatorname{Ext}_{A_{n}}(\mathbf{Z} / 2, \mathbf{Z} / 2)$ defined above,

$$
r_{k}\left(v_{j}^{2^{m}}\right)= \begin{cases}v_{j}^{2^{m}} / K_{n} & \text { if } k=0, \\ v_{j-1}^{2^{m}} / K_{n} & \text { if } k=2^{m+j-n-1}, \\ \text { Zero } / K_{n} & \text { otherwise. }\end{cases}
$$


PROOF. There is a version of the ring homomorphism $r$ above defined for the Hopf algebra $A / / E_{n}$ given by the formula of Lemma 4.1 for the dual $\left(A / / E_{n}\right)^{*}$. This induces in Ext:

$$
r: \operatorname{Ext}_{E_{n}}(\mathbf{Z} / 2, \mathbf{Z} / 2) \rightarrow \underset{m \geqslant 0}{\bigoplus} \operatorname{Ext}_{E_{n-1}}\left(\Sigma^{m 2^{n+1}} \mathbf{Z} / 2, \mathbf{Z} / 2\right)
$$

just as in (4.2). Now the bar construction for calculating $\operatorname{Ext}_{E_{n}}$ begins:

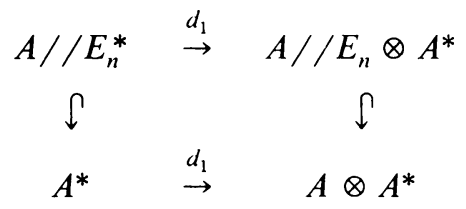

Here $d_{1}\left(\zeta_{n}\right)=\sum_{i=1}^{n}\left[\zeta_{n-1}^{2^{i}}\right] \zeta_{i}$ which corresponds to $v_{n} \in \operatorname{Ext}_{E_{n}}(\mathbf{Z} / 2, \mathbf{Z} / 2)$, where the $i=0$ term vanishes. So

$$
\begin{aligned}
d_{1}\left(\zeta_{n}\right) & =d_{i}\left(\zeta_{n}+\zeta_{n-1} t^{2^{n}}\right) \text { since } d_{1} \text { is natural w.r.t. the map } r \text { induced in } A / / E_{n}^{*} \\
& =d_{1}\left(\zeta_{n}\right)+d_{1}\left(\zeta_{n-1}\right) t^{2^{n}},
\end{aligned}
$$

which corresponds to $v_{n}+v_{n-1} t^{2^{n}}$. So in $\operatorname{Ext}_{E_{n}}$, we have $r\left(v_{n}\right)=v_{n}+v_{n-1} t^{2^{n}}$. Extending this to $v_{n}^{2^{m}}$, and looking at the corresponding map in $\operatorname{Ext}_{A_{n}}$ completes the proof.

5. Proof of the main theorem. In this section, we prove Theorem $C$ and derive Corollary D from it. The proof is to some extent a simplified version of Johnson and Yosimura's proof that in a $\mathrm{BP}_{*} \mathrm{BP}$-comodule $M$, elements that are $v_{n}$-torsion are also $v_{k}$-torsion, for $0 \leqslant k \leqslant n$ [9]. Our operations

$$
r_{j}: \operatorname{Ext}_{A_{k}}(\mathbf{Z} / 2, \mathbf{Z} / 2) \rightarrow \operatorname{Ext}_{A_{k-1}}\left(\Sigma^{j 2^{k+1}} \mathbf{Z} / 2, \mathbf{Z} / 2\right)
$$

replace the Quillen operations of BP-theory.

We recall the statement of our main theorem.

TheOREM C. If $x \in \operatorname{Ext}_{A}^{s, t}(\mathbf{Z} / 2, \mathbf{Z} / 2)$ is $v_{n}$-periodic, then $x$ is also $v_{n+k}$-periodic for all $k \geqslant 0$.

Equivalently, if $x \in \mathrm{Ext}_{A}^{s, t}(\mathbf{Z} / 2, \mathbf{Z} / 2)$ is $v_{n}$-torsion, then $x$ is also $v_{k}$-torsion for all $k$ such that $0 \leqslant k \leqslant n$.

Proof of Theorem C. Let $x \in \operatorname{Ext}_{A}(\mathbf{Z} / 2, \mathbf{Z} / 2)$ be $v_{n}$-torsion. Then for all $k$ sufficiently large, $q_{k}(x)=\hat{x}$ is $v_{n}$-torsion in $\operatorname{Ext}_{A_{k}}(\mathbf{Z} / 2, \mathbf{Z} / 2)$. Since $x \in$ $\operatorname{Ext}_{A}(\mathbf{Z} / 2, \mathbf{Z} / 2), r_{0}(\hat{x})=r_{0}\left(q_{k}(x)\right)=p_{k-1}(\hat{x})=q_{k-1}(x)$, and $r_{m}(\hat{x})=0$ for $m>0$, by the remarks following (4.4). Recall that $v_{n}^{s}$ is a coset. As before, let $K_{k}$ denote the kernel of the restriction map $\operatorname{Ext}_{A_{k}} \rightarrow \operatorname{Ext}_{E_{k}}$ (so that $K_{k}$ is bigraded). Let $\bar{v}_{n}^{s}$ be a fixed represenatative for the coset, $v_{n}^{s}$. Then any element in the coset can be represented as $\bar{v}_{n}^{s}+y$, for $y \in K_{n}$. Then $x$ being $v_{n}$-torsion implies that

$$
\left[\prod_{y \in K}\left(\bar{v}_{n}^{s}+y\right)\right]^{t} \cdot \hat{x}=0, \quad \text { for some } t .
$$


For all $m \in \mathbf{N}$, then

$$
r_{m}\left[\left[\prod_{y \in K}\left(\nabla_{n}^{s}+y\right)\right]^{t} \cdot \hat{x}\right]=r_{m}\left(\prod_{y \in K}\left(\nabla_{n}^{s}+y\right)^{t}\right)-q_{k-1}(x)=0 .
$$

For the appropriate value of $m$ (given in 4.5), this becomes

$$
\left[\prod_{z \in K}\left(\bar{v}_{n-1}^{s}+z\right)^{t}\right] \cdot q_{k-1}(x)=0
$$

where the classes $z$ are in $K_{k-1}$ and $r_{m}\left(\bar{v}_{n}^{s}\right)$ is a particular element $\bar{v}_{n-1}^{s}$ mapping to the appropriate class in $\operatorname{Ext}_{E_{k-1}}$. This implies that $\left[\prod_{w \in K_{k-1}}\left(\bar{v}_{n-1}^{s}+w\right)^{t^{\prime}}\right] \cdot q_{k-1}(x)$ $=0$. This shows that $q_{k-1}(x)$ is $v_{n-1}$-torsion, completing the proof.

From this, we prove

\section{COROLlaRY D. There is a chromatic filtration}

$$
\operatorname{Ext}_{A}(\mathbf{Z} / 2, \mathbf{Z} / 2)=F_{-1} \supset F_{0} \supset F_{1} \supset \cdots \supset F_{i} \supset \cdots
$$

such that $F_{i}-F_{i-1}$ is the set of classes that are $v_{i+1}$-periodic but $v_{k}$-torsion for all $k \leqslant i$.

Proof. Recall that $V_{i}^{s, t}=\lim \left[\operatorname{Ext}_{A_{k}}^{s, t}(\mathbf{Z} / 2, \mathbf{Z} / 2)\left(v_{i}^{-1}\right)\right]$, and that the map $f_{i}$ : $\operatorname{Ext}_{A}^{s, t}(\mathbf{Z} / 2, \mathbf{Z} / 2) \rightarrow V_{i}^{s, t}$ defines the $v_{i}$-torsion and periodic classes in $\operatorname{Ext}_{A}^{s, t}(\mathbf{Z} / 2, \mathbf{Z} / 2)$. Define $F_{i}$ to be the kernel of the map $f_{i}$ for all $i \geqslant 0 . F_{i}$ contains $F_{i+1}$ by Theorem C. Defining $F_{-1}$ to be all of $\operatorname{Ext}_{A}(\mathbf{Z} / 2, \mathbf{Z} / 2)$ completes the proof.

\section{REFERENCES}

1. J. F. Adams, A periodicity theorem in homological algebra, Proc. Cambridge Philos. Soc. 62 (1966), $365-377$.

2. On the groups $J(X)$. IV, Topology 5 (1966), 21-71.

3. N. A. Baas, On the bordism theory of manifolds with singularity, Math. Scand. 33 (1973), 279-302.

4. M. G. Barratt, Mimeographed notes, Seattle conferences, 1963.

5. E. H. Brown and F. P. Peterson, $A$ spectrum whose $\mathbf{Z}_{p}$ cohomology is the algebra of reduced powers, Topology 5 (1966), 149-154.

6. D. M. Davis and M. E. Mahowald, $v_{1}-$ and $v_{2}$-periodicity in stable homotopy theory, Amer. J. Math. 103 (1981), 615-659.

7. Ext over the subalgebra $A_{2}$ of the Steenrod algebra for stunted projected spaces, Canad. Math. Soc. 2 (1982), 297-342.

8. P. J. Hilton and U. Stammbach, A course in homological algebra, Springer-Verlag, Berlin and New York, 1970.

9. D. C. Johnson and Z. Yosimura, Torsion in Brown-Peterson homology and Hurewicz homomorphisms, Osaka J. Math. 17 (1980), 117-136.

10. M. E. Mahowald, The primary $v_{2}$-periodic family, Math. Z. 177 (1981), 381-393.

11. W. Lellmann and M. E. Mahowald, Some generalizations of the lambda algebra, Math. Z. 192 (1986), 243-251.

12. W.-H. Lin, Cohomology of sub-Hopf-algebras of the Steenrod algebra, J. Pure Appl. Algebra 10 (1977), 101-113.

13. H. R. Miller, D. Ravenel and W. S. Wilson, Periodic phenomena in the Adams-Novikov spectral sequence, Ann. of Math. (2) 106 (1977), 469-516.

14. F. P. Peterson, Lectures on cobordism theory, Kinikuniya Bookstore, Tokyo, Japan, 1968. 
15. P. L. Shick, Thesis, Northwestern University, 1984.

16. N. E. Steenrod and D. B. A. Epstein, Cohomology operations, Ann. of Math. Studies, no. 50, Princeton Univ. Press, Princeton, N. J., 1962.

17. M. C. Tangora, On the cohomology of the Steenrod algebra, Math. Z. 116 (1970), 18-64.

18. C. Wilkerson, The cohomology algebras of finite dimensional Hopf algebras, Trans. Amer. Math. Soc. 264 (1981), 137-150.

19. A. Liulevicius, Cohomology of Massey-Peterson algebras, Math. Z. 105 (1968), 226-256.

Department of Mathematics, Northwestern University, Evanston, Illinois 60201 (Current address of Mark Mahowald)

Department of Mathematics, John Carroll University, Cleveland, Ohio 44116

Current address (Paul Shick): Department of Mathematics, University of Washington, Seattle, Washington 98195 